\title{
Modulation of Cardiac Autonomic Dysfunction in Ischemic Stroke following Ayurveda (Indian System of Medicine) Treatment
}

\author{
Sriranjini Sitaram Jaideep, ${ }^{1,2}$ Dindagur Nagaraja, ${ }^{3}$ Pramod Kumar Pal, ${ }^{3}$ \\ D. Sudhakara, ${ }^{4}$ and Sathyaprabha N. Talakad ${ }^{1}$ \\ ${ }^{1}$ Department of Neurophysiology, National Institute of Mental Health \& Neurosciences (NIMHANS), Hosur Road, \\ Bangalore 560029, India \\ ${ }^{2}$ Clinical Research Group, School of Health Sciences, Institute of Transdisciplinary Health Sciences and Technology (FRLHT-ITDHST), \\ Bangalore 560106, India \\ ${ }^{3}$ Department of Neurology, National Institute of Mental Health \& Neurosciences (NIMHANS), Hosur Road, Bangalore 560029, India \\ ${ }^{4}$ Advanced Center for Ayurveda in Mental Health and Neurosciences, National Institute of \\ Mental Health \& Neurosciences (NIMHANS), Hosur Road, Bangalore 560029, India
}

Correspondence should be addressed to Sathyaprabha N. Talakad; drsathyaprabha@gmail.com

Received 4 December 2013; Accepted 2 April 2014; Published 26 May 2014

Academic Editor: Joen-Rong Sheu

Copyright (c) 2014 Sriranjini Sitaram Jaideep et al. This is an open access article distributed under the Creative Commons Attribution License, which permits unrestricted use, distribution, and reproduction in any medium, provided the original work is properly cited.

\begin{abstract}
Objectives. Cardiac autonomic dysfunction in stroke has implications on morbidity and mortality. Ayurveda (Indian system of medicine) describes stroke as pakshaghata. We intended to study the effect of Ayurveda therapies on the cardiac autonomic dysfunction. Methods. Fifty patients of ischemic stroke (middle cerebral artery territory) (mean age $39.26 \pm 9.88$ years; male 43 , female 7) were recruited within one month of ictus. All patients received standard allopathic medications as advised by neurologist. In addition, patients were randomized to receive physiotherapy (Group I) or Ayurveda treatment (Group II) for 14 days. Continuous electrocardiogram and finger arterial pressure were recorded for $15 \mathrm{~min}$ before and after treatments and analyzed offline to obtain heart rate and blood pressure variability and baroreflex sensitivity (BRS). Results were analysed by RMANOVA. Results. Patients in Group II showed statistically significant improvement in cardiac autonomic parameters. The standard deviation of normal to normal intervals, and total and low frequency powers were significantly enhanced $(F=8.16, P=0.007, F=9.73, P=0.004$, $F=13.51$, and $P=0.001$, resp.). The BRS too increased following the treatment period $(F=10.129, P=0.004)$. Conclusions. The current study is the first to report a positive modulation of cardiac autonomic activity after adjuvant Ayurveda treatment in ischemic stroke. Further long term studies are warranted.
\end{abstract}

\section{Introduction}

Stroke leads to dysfunction of the autonomic nervous system, including cardiovascular, gastrointestinal, genitourinary, thermoregulatory, and sudomotor systems [1]. The cardiovascular regulation is affected due to aberrant activity in the central areas regulating these functions or their pathways [2]. This has been associated with fatal complications [3]. Heart rate variability (HRV), blood pressure variability
(BPV), and baroreflex sensitivity (BRS) have been identified as subtle markers of such cardiac dysregulation, persisting in the chronic state and associated with poor prognosis [4-8]. Effective strategies to modulate cardiac autonomic dysfunction may hence provide better prognosis and survival. The possibility of enhancing the baroreflex as a therapeutic strategy in acute stroke has been proposed [9]. Though $\beta$ blockers can influence baroreflex by vagal modulation [10], they are associated with potential harm [11, 12]. 
From an Ayurveda perspective, stroke is recognized as pakshaghata (hemiplegia), a disease attributed to an aberration of vatadosha (a physiologic entity). Cardiac dysregulation is understood as a secondary consequence of this process [13]. The diverse treatments advocated in Ayurveda for this disease primarily harmonize the aberrant physiology. Hence, we hypothesized that the Ayurveda therapy may also modulate the cardiac dysregulation. The current study aimed to evaluate the effect of adjuvant Ayurveda therapies on cardiac dysregulation in ischemic stroke using HRV, BPV, and BRS parameters.

\section{Patients and Methods}

The present study was conducted in the Departments of Neurophysiology, Neurology and Advanced Center for Ayurvedic Research in Mental health and Neurosciences, National Institute of Mental Health and Neuro Sciences (NIMHANS), Bangalore. The study was a comparative randomized clinical evaluation, approved by the Institute Ethics Committee, Advanced Center for Ayurvedic Research in Mental health and Neurosciences, NIMHANS. Patients and/or relatives were explained about the nature and design of the study and informed consent was obtained.

2.1. Inclusion and Exclusion Criteria. Patients diagnosed with ischemic stroke by a neurologist and as pakshaghata (hemiplegia) by an Ayurveda physician by thorough clinical examination and imaging were screened from casualty section, stroke unit, and neurorehabilitation section, NIMHANS. Patients were included if aged between 20 and 60 years, of either gender; with first time ischemic stroke in middle cerebral artery (MCA) territory diagnosed by history, clinical examination and CT scan/MRI of brain, between 10 and 30 days of stroke, with stable neurological status following the necessary acute phase treatment after stroke, motor weakness of at least one limb of MRC Grade 0-3 and fulfilling the criteria to undergo Ayurveda therapies. Patients were excluded if sensorium was altered or severely aphasic to comprehend simple instructions, associated with comorbidities such as uncontrolled hypertension, diabetes, or other systemic diseases, pregnant or lactating. All patients were admitted in the Advanced Ayurvedic Research Institute for Mental Health and Neurosciences, NIMHANS, and randomized to receive the following for a period of 15 days.

2.2. Intervention. Group I received conventional treatment including antiplatelet agents or anticoagulants, antihypertensives, and medications for diabetes mellitus hyperlipidemia along with physiotherapy and speech therapy as required. Group II received conventional treatment and speech therapy as in Group I. In addition they received adjuvant Ayurveda treatment consisting of (a) external therapies such as Abhyanga (methodical massage) with Niramisha mahamasha taila and Bhashpa svedana (steam therapy) from day 1 to day 14, Matra basti with $60 \mathrm{~mL}$ of Balaswagandhadi taila from day 8-14 and (b) internal medication of Ashtavarga kashaya orally, $15 \mathrm{~mL}$ TID with $15 \mathrm{~mL}$ warm water and
Ksheerabala 101 orally, 5 drops BD with $15 \mathrm{~mL}$ warm water. Refer Table 3 for details of medicine used.

2.3. Assessment Criteria. Patients were evaluated with cardiac autonomic function tests which included heart rate variability (HRV), blood pressure variability (BPV), and baroreflex sensitivity (BRS), before and after the treatment. The resting heart rate (HR) and blood pressure (BP) recordings were carried out in the autonomic laboratory, Department of Neurophysiology, NIMHANS under standardized conditions $[14,15]$. Patients were advised to abstain from alcohol and nicotine for 24 hours before evaluation. The tests were performed in a silent room maintained at a temperature of $22-26^{\circ} \mathrm{C}$, between 8 am and $11 \mathrm{am}$. Patients were advised to have light breakfast two hours prior to the tests and empty bowel and bladder before the tests. They were familiarized with the laboratory settings and briefed about the tests. Recordings were done after 30 minutes of supine rest.

2.3.1. Resting HRV. Lead II electrocardiogram (ECG) and breathing signals were conveyed through analog digital converter (Power lab, 16 channels data acquisition system, $\mathrm{AD}$ Instruments, Australia) with a sampling rate of $1024 \mathrm{~Hz}$. MLS 310 Module was used to analyze the different HRV measures. It was ensured that patients were breathing at normal respiratory rate of $12-15$ breaths/min by recording the respiratory movements using stethograph. The data were stored in PC and analyzed offline using an automatic programme that allowed visual checking of the raw ECG and breathing signals. Fifteen-minute basal recordings were stored and later, a 5minute artifact/ectopic free segment was analyzed to obtain time domain and frequency domain parameters of HRV as per the guidelines of task force report [16].

2.3.2. Resting BPV and Spontaneous BRS. Blood pressure was recorded using the Finometer (Finapres Medical Systems (FMS), The Netherlands). Following height correction, physiological and return to flow calibrations, the finger arterial pressure was recorded continuously for $15 \mathrm{~min}$. The Physical was then turned off during the recording. The recorded data was downloaded offline using Finolink software provided by FMS and stored in a PC. All recordings were scanned and 5-minute artifact/ectopic free segment was analyzed using Nevrokard cardiovascular parameter analysis (CVPA) software (version 2.1.0) to obtain the time domain and frequency domain parameters of blood pressure variability. Baroreflex sensitivity (BRS) values were derived by sequence and spectral methods using the same software as per guidelines $[17,18]$.

2.4. Control Data. To assess the presence and extent of cardiac autonomic dysfunction in the ischemic stroke patients, similar ECG data was obtained from 30 clinically healthy subjects.

\subsection{Description of Ayurveda Therapies}

2.5.1. Abhyanga (Therapeutic Massage). Abhyanga was performed during morning hours ( $8 \mathrm{am}-12$ noon). Patient was 
TABLE 1: Demographic details of the patients.

\begin{tabular}{lccc}
\hline Variable & Group I $(n=25)$ & Group II $(n=25)$ & $P$ value \\
\hline Age (in years) & $40.12 \pm 9.31$ & $38.40 \pm 10.50$ & 0.543 \\
Gender $(\mathrm{M}: \mathrm{F})$ & $20: 5$ & $23: 2$ & 0.111 \\
Height $(\mathrm{cm})$ & $168.08 \pm 2.04$ & $67.01 \pm 2.51$ & 0.274 \\
Weight $(\mathrm{kg})$ & $66.72 \pm 2.38$ & $23.33 \pm 0.76$ & 0.936 \\
BMI $\left(\mathrm{kg} / \mathrm{m}^{2}\right)$ & $24.04 \pm 0.78$ & $17.20 \pm 5.74$ & 0.519 \\
Duration of illness (days) & $14.96 \pm 4.16$ & 17 & 0.121 \\
Smoking & 14 & 17 & 0.718 \\
Alcohol & 15 & 3 & 0.860 \\
Hypertension & 2 & 2 & 1.000 \\
Diabetics & 2 & 8 & 1.000 \\
Right side infarct & 10 & 17 & - \\
Left side infarct & 15 & - \\
\hline
\end{tabular}

M: male; F: female; BMI: body mass index; values expressed as mean \pm SD.

advised to empty bowel and bladder before starting the therapy. Patient was made to lie in supine posture over the Droni (Abhyanga table). Requisite quantity of Niramisha Mahamasha taila (medicated oil) was warmed on a water bath and applied on the patient's body. The entire body was then massaged by 2 masseurs with uniform pressure using long strokes and in circular motion, as required [19].

2.5.2. Svedana (Sudation). Bhashpa sveda variety of Svedana was done. The patient was made to sit in a Bhashpa svedana yantra (customized wooden box) into which warm vapors were passed. This induced perspiration in the patient. This was continued for $10-15 \mathrm{~min}$ depending on the patients' tolerance level [19].

2.5.3. Basti (Therapeutic Enema). Matra basti (therapeutic oil enema) variety of basti was used. The procedure was performed after noon (between 12 noon and $3 \mathrm{pm}$ ). Patients were advised to take light food and empty bowel and bladder before starting the therapy. Local Abhyanga and svedana was performed around the gluteal region. Following this, the patient was advised to lie down in the left lateral position on Abhyanga table with left led stretched out and the right leg flexed at the knee and hip joints. The patient's left arm was placed beneath his head. A pinch of Saindhava lavana (black salt) was mixed in $60 \mathrm{~mL}$ of lukewarm Balaswagandhadi taila and drawn into a syringe. A rubber catheter was attached to enema syringe and its tip was lubricated with the same oil. The catheter was then introduced into the anus (up to 4 inches length). Patient was advised to breathe slowly and deeply and the oil was steadily pushed into the rectum. The patient was then made to lie supinely while his legs were raised at the hips for 3-4 times and the gluteal region was massaged gently for few seconds. Patient was advised to retain the enema as long as possible [19].

2.6. Statistical Methods. Statistical analysis was performed using SPSS. Chi-square and Fisher Exact test was used to find the significance of categorical data comparison between two groups of patients. Independent sample " $t$ " test was carried out to compare (a) the HRV variables between pakshaghata patients and healthy subjects and (b) the baseline characteristics of the two groups of patients. $2 \times 2$ repeated measures analysis of variance (RMANOVA) was used to find the significance of study parameters within and between the two groups of patients. The two independent factors included in the analysis were (1) treatment (between-subjects factor) with two levels: conventional treatment with physiotherapy and conventional treatment with Ayurveda and (2) time (withinsubjects factor) with two levels: baseline and after 2 weeks. The main effects when found to be significant were further tested for the direction of differences by using pairwise comparisons adjusted by Bonferroni's method. Values are represented as mean \pm SEM and significance was considered if $P<0.05$.

\section{Results}

3.1. Demographic Details. Fifty patients satisfying the study criteria were recruited and allotted to receive treatment. Baseline evaluation of the demographic details of patients indicated the homogeneity in age, gender, height, weight, body mass index, risk factors predisposing to stroke, duration of illness, and side of infarct in both the groups (Table 1). Two patients in Group II were unable to stay in the hospital for the entire duration of treatment and hence dropped from the study. HRV could not be analyzed in 12 patients (Group I-8, Group II-4) due to the presence of artifacts in the ECG. BPV and BRS analysis was done in a subsample of 24 cases (Group I-11, Group II-13).

3.2. Comparison of Ischemic Stroke Patients with Healthy Controls. At baseline, the stroke patients demonstrated significantly lower mean NN and higher heart rate when compared with healthy controls. In addition, the time domain measures SDNN and RMSSD and frequency domain measures TP, LF, $\mathrm{HF}$, and HFnu were significantly reduced and the LF/HF ratio was significantly higher (Table 2). 
TABLE 2: Comparison of heart rate variability (HRV) measures between healthy controls and ischemic stroke patients.

\begin{tabular}{lccc}
\hline HRV variables & Controls $(n=30)$ & Patients $(n=38)$ & $P$ value \\
\hline NN interval $(\mathrm{ms})$ & $869.96 \pm 23.15$ & $795.67 \pm 16.22$ & $0.009^{* *}$ \\
HR $(\mathrm{bpm})$ & $70.44 \pm 1.88$ & $76.08 \pm 1.61$ & $0.026^{*}$ \\
SDNN $(\mathrm{ms})$ & $48.01 \pm 3.42$ & $36.47 \pm 2.25$ & $0.004^{* *}$ \\
RMSSD $(\mathrm{ms})$ & $38.06 \pm 4.13$ & $28.00 \pm 2.80$ & $0.027^{*}$ \\
TP $\left(\mathrm{ms}^{2}\right)$ & $2489.00 \pm 337.06$ & $1286.63 \pm 148.50$ & $0.000^{* * *}$ \\
LF $\left(\mathrm{ms}^{2}\right)$ & $527.16 \pm 62.53$ & $290.11 \pm 34.87$ & $0.001^{* *}$ \\
LFnu & $44.92 \pm 2.71$ & $49.01 \pm 3.13$ & 0.481 \\
HF $\left(\mathrm{ms}^{2}\right)$ & $812.62 \pm 196.77$ & $364.71 \pm 90.81$ & $0.005^{* *}$ \\
HFnu & $49.18 \pm 2.61$ & $41.87 \pm 3.30$ & $0.042^{*}$ \\
Sympathovagal balance & $1.09 \pm 0.13$ & $1.66 \pm 0.20$ & $0.043^{*}$ \\
\hline
\end{tabular}

NN: R-R interval (ms); HR: heart rate (bpm: beats per minute); SDNN: standard deviation of NN intervals (ms); RMSSD: square root of the mean of the sum of squares of differences between adjacent NN intervals (ms); TP: total power $\left(\mathrm{ms}^{2}\right)$; LF: low frequency power $\left(\mathrm{ms}^{2}\right)$; HF: high frequency power $\left(\mathrm{ms}^{2}\right)$; nu: normalized units; SVB: sympathovagal balance; values expressed as mean \pm SEM; ${ }^{*} P<0.05,{ }^{* *} P<0.01,{ }^{* * *} P<0.001$.

3.3. Effect of Treatments on HRV Measures. Significant treatment effect over time was observed for $\operatorname{SDNN}(F=8.16, P=$ $0.007)$, TP $(F=9.73, P=0.004)$, and $\mathrm{LF}$ power $(F=13.51$, $P=0.001)$. Adjusted pairwise comparisons for time showed improvement in these parameters in the group treated with adjuvant Ayurveda therapies. There was also a significant group effect for mean $\mathrm{NN}(F=6.41, P=0.016)$, $\operatorname{HR}(F=5.88, P=0.021)$, and $\operatorname{RMSSD}(F=5.87, P=$ $0.021)$ and adjusted pairwise comparison for group was significant for RMSSD in the group treated with adjuvant Ayurveda therapies at 2 weeks $(P=0.025)$. The LF/HF ratio showed marginal group effect $(F=3.34, P=0.076)$. Pairwise adjustment for group comparisons indicated marginally lesser LF/HF ratio $(P=0.067)$ in the patients treated with adjuvant Ayurveda therapies at 2 weeks. Though there was increase in LF power, no significant change in LFnu or $\mathrm{LF} / \mathrm{HF}$ ratio in the group treated with adjuvant Ayurveda therapies (Figures 1(a)-1(f)).

3.4. Effect of Treatments on BPV and BRS Measures. The SBP, DBP, and MBP showed a trend for decrease in both the groups but it was not statistically significant. We also did not find any significant changes in the time domain and frequency domain measures of BPV following treatment in both the groups (data not shown). Among the sequence and spectral parameters of spontaneous baroreflex, the Up BRS was significantly enhanced in patients treated with adjuvant Ayurveda therapies after pairwise adjustment for time and group at 2 weeks (Figure $1(\mathrm{~g})$ ).

\section{Discussion}

The science of Ayurveda has evolved by in-depth observation and experience of scholars. However, the potential benefits of this science need to be evaluated with objectivity. The current study demonstrated that cardiac autonomic functions can be used as a marker to detect one facet of aberrant physiology in pakshaghata (hemiplegia) and that a whole system Ayurveda treatment protocol as used here was able to modulate the same.
To the best of our knowledge, this is the first study to evaluate the effect of adjuvant Ayurveda therapies on cardiac autonomic dysfunction in ischemic stroke. The study has used a whole system treatment protocol, adapted from Ayurveda classics keeping in mind convenience for clinical application. This is a positive deviation from single drug clinical trial modules that may be ineffective to capture the essence of Ayurveda treatments [20]. However, we were limited by factors including short follow-up period that prevented evaluation of lasting effect of the treatment. Also, HRV could not be analyzed in few patients $(n=12)$ due to the presence of artifacts. BPV and BRS analysis was done in a subsample of 24 cases. However, compared to the total sample, the subsample analysis was not significantly different indicating that subsample is representative of the total sample.

In the current study, when compared with healthy controls, the patients with ischemic stroke demonstrated significantly lower mean NN and higher heart rate, time domain measures (SDNN, RMSSD) and frequency domain measures (TP, LF, HF, and HFnu) were reduced, and the LF/HF ratio was significantly higher indicating definite cardiac autonomic dysfunction. These findings of altered autonomic activity with increased sympathetic activation correlate with earlier studies on ischemic stroke $[6,14,21,22]$. The aberrant neurocardiac regulation persists even in the postacute phase [23] and abnormal heart rate dynamics has also been associated with poststroke mortality [24].

Following intervention, it was observed that the patients receiving adjuvant Ayurveda therapies demonstrated significant enhancement of time domain (SDNN) and frequency domain parameters (TP, LF power) of HRV. There was also a significant group effect for mean NN and RMSSD and adjusted pairwise comparison for group was significant in the group treated with adjuvant Ayurveda therapies. This combined with a trend for increase of the HF power and a marginally lesser LF/HF in this group. Though there was increase in LF power, no significant change was observed in LFnu or LF/HF ratio. Among the BPV measures, the SBP, DBP, and MBP showed a trend for decrease in both the groups but it was not statistically significant. We also did not 


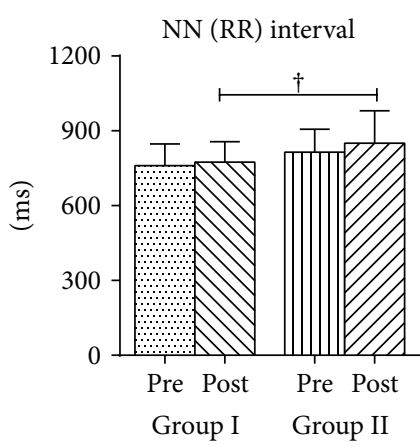

(a)

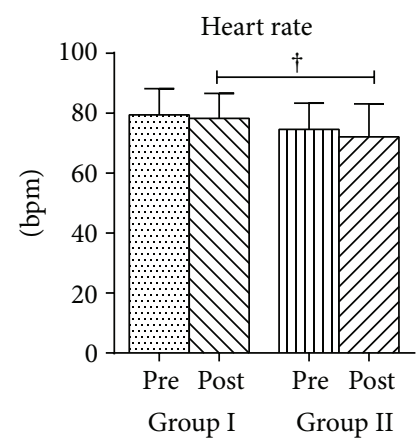

(b)

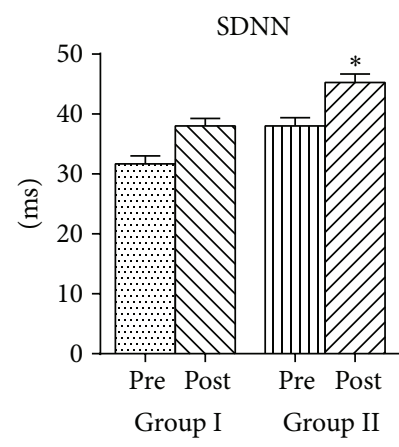

(c)

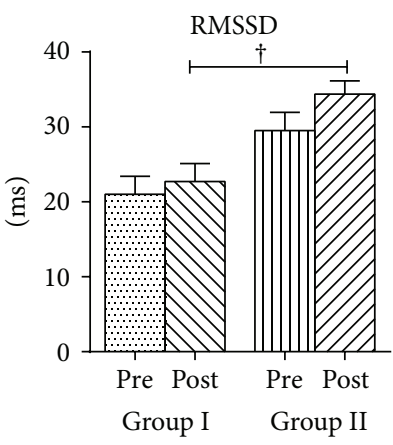

(d)

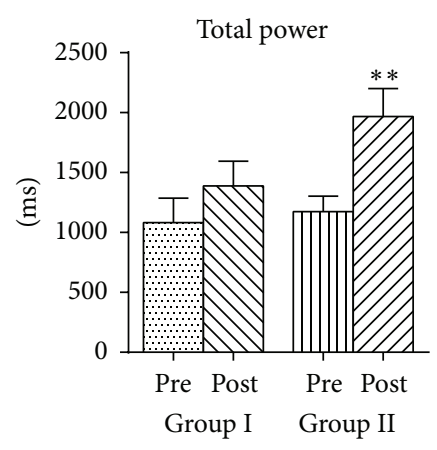

(e)

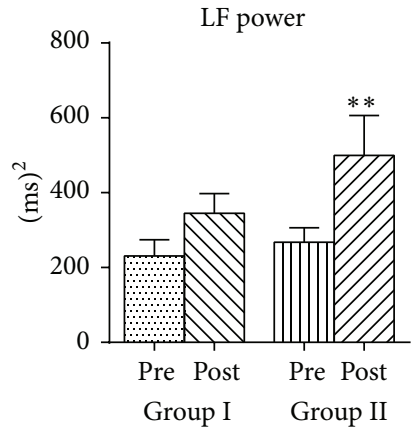

(f)

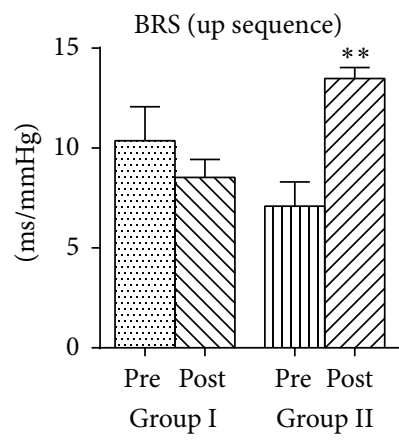

(g)

FIGURE 1: Effect of intervention on heart rate variability measures and baroreflex sensitivity (a) NN-R-R interval (ms); (b) HR-heart rate (bpm-beats per minute); (c) SDNN_standard deviation of NN intervals (ms); (d) RMSSD—square root of the mean of the sum of squares of differences between adjacent NN intervals (ms); (e) TP-total power $\left(\mathrm{ms}^{2}\right)$; (f) LF-low frequency power (ms $\left.{ }^{2}\right)$; (g) BRS—baroreflex sensitivity $(\mathrm{ms} / \mathrm{mm} \mathrm{Hg})$; values expressed as Mean \pm SEM; ${ }^{*} P<0.05,{ }^{* *} P<0.01$, pre versus post, within group comparison; ${ }^{\dagger} P<0.05$ Group I versus Group II at 2 weeks.

find any significant changes in the other BPV parameters following treatment in both the groups. Among the sequence and spectral parameters of spontaneous baroreflex, the Up BRS was significantly enhanced and the $\alpha \mathrm{HF}$ also showed a trend for increase following adjuvant Ayurveda therapies.

The time domain measure SDNN and frequency domain measure of TP are robust indicators of overall variability and reflect vagal activation. RMSSD too mirrors the parasympathetic influence on the HRV $[16,25]$. The physiological implications of LF power have been conflicting. Earlier studies have demonstrated LF power to be an indicator of sympathetic tone largely $[16,26,27]$ while a parasympathetic contribution is also hypothesized [28]. Recent work in this area, however, suggests that the LF component of HRV reflects the baroreflex gain and hence interventions that increase the LF power may modulate the baroreflexes in turn affecting the cardiac autonomic outflow [29-31]. It is also debated that reduced LF power can exist despite clinically evident sympathetic overactivity and this can be attributed to a reduced baroreflex modulation amongst others [32]. In our study too, it was observed that the LF power of the ischemic stroke patients was significantly lower than the healthy controls at baseline, indicating an initially reduced baroreflex function. However, the subsequent increase in most of the HRV components compounded by the increased BRS following adjuvant Ayurveda therapies indicates a positive cardiac autonomic regulation amongst others.

In the present study, we used a whole system approach combining different modes of treatment as mentioned in Ayurveda. These treatments are indicated for neurological illnesses generally and pakshaghata (hemiplegia) specifically. Most of the herbs used as internal medication in the current study have been studied for their antioxidant and neuroprotective activity including Bala (Sida cordifolia) in Ksheerabala 101 [33, 34], Devadaru (Cedrus deodara) [35], Lashuna (Allium cepa) [36, 37], Shunti (Zingiber officinale) [38], and so forth. The release of free radicals in the brain is well established in acute and chronic stages of ischemic stroke $[39,40]$ and antioxidant therapy is seen to be beneficial in late treatment of ischemic stroke too [41]. The therapeutic procedures included Abhyanga (methodical massage), Svedana (sudation), and Basti (colonic administration. Different massage techniques are known to enhance vagal activity $[42,43]$. A study on oriental massage demonstrated an improved profile of cardiovascular autonomic regulation [44]. The benefits of svedana (sudation) may be akin to that of thermal therapy in the saunas. It is observed that repeated sauna improves cardiac function and hemodynamics [45]. Parasympathetic activity is enhanced and sympathetic activity also reduced on HRV analysis following application of heat and steam generating (HSG) sheets [46, 47]. 
TABLE 3: Medicines used.

\begin{tabular}{lc}
\hline & \multicolumn{1}{c}{ Ingredients } \\
Sanskrit name & Latin name \\
\hline Bala & Sida cordifolia \\
Sahachara & Barleria prionitis \\
Eranda & Ricinus communis \\
Shunti & Zingiber officinale \\
Rasna & Vanda roxburghii \\
Suradruma & Cedrus deodara \\
Sinduvara & Vitex negundo \\
Lasuna & Allium cepa \\
\hline & (2) Ksheerabala 101 [49] \\
Balamoola & Sida cordifolia \\
Tila taila & Gingelly oil \\
Goksheera & Milk \\
Balamoola kalka & Sida cordifolia
\end{tabular}

(3) Balaswagandhadi thailam [50]

\begin{tabular}{ll} 
Ashwagandha & Withania somnifera \\
Bala & Sida cordifolia \\
Tila taila & Gingelly oil \\
Lodhra & Symplocos racemosa \\
Sarjikakshara & \\
Bidara & Zizyphus jujuba \\
Haridra & Curcuma longa \\
Daruharidra & Berberis aristata \\
Renuka seeds & Vitex negundo \\
Kushta & Saussurea lappa \\
Musta & Cyperus rotundus \\
Srigandha & Santalum album \\
Sariva & Hemidesmus indicus \\
Katukarohini & Piccrorhiza kurro \\
Shatahva & Anethum sowa \\
Devadaru & Cedrus deodara \\
Manjistha & Rubia cordifolia \\
Yashti & Glycyrrhiza glabra \\
Usheera & Vetiveria zizanioides \\
Ksheera & Milk \\
\hline
\end{tabular}

(4) Niramisha mahamasha taila [51]

\begin{tabular}{ll} 
Masha & $\begin{array}{l}\text { Phaseolus mungo } \\
\text { Bilva }\end{array}$ \\
Aggnimantha marmelos \\
Shyonaka & Clerodendron latifolium \\
Kashmiri & Oroxylum indicum \\
Patala & Gmelina arborea \\
Brihati & Stereospermum suaveolens \\
\hline
\end{tabular}

TABle 3: Continued.

\begin{tabular}{|c|c|}
\hline \multicolumn{2}{|c|}{ Ingredients } \\
\hline Sanskrit name & Latin name \\
\hline Kantakari & Solanum indicum \\
\hline Gokshura & Tribulus terrestris \\
\hline Salaprni & Desmodium gangeticum \\
\hline Prishnaparni & Uraria picta \\
\hline Jala & Water \\
\hline Tila taila & Gingelly oil \\
\hline Ksheera & Milk \\
\hline Ashwagandha & Withania somnifera \\
\hline Vidari & Ipomea digitata \\
\hline Shatavari & Asparagus racemosus \\
\hline Kachora & Hedychium spicatum \\
\hline Devadaru & Cedrus deodara \\
\hline Bala & Sida cordifolia \\
\hline Rasna & Vanda roxburghii \\
\hline Prasarini & Paederia foetida \\
\hline Kushta & Saussurea lappa \\
\hline Shatahva & Anethum sowa \\
\hline Parushaka & Grewia asiatica \\
\hline Bharangi & Clerodendrum serratum \\
\hline Punarnava & Boerhavia diffusa \\
\hline Pippali Mula & Piper longum \\
\hline Chitrak & Plumbago zeylanica \\
\hline Jivanti & Leptadenia reticulata \\
\hline Sariva & Hemidesmus indicus \\
\hline Mashaparni & Teramnus labialis \\
\hline Mudgaparni & Phaseolus trilobus \\
\hline Guduchi & Tinospora cordifolia \\
\hline Yashti & Glycyrrhiza glabra \\
\hline Saindhava lavana & Rock salt \\
\hline Hingu & Ferula foetida \\
\hline
\end{tabular}

A probable mode of action of the therapies involved has been put forth by way of evidence that we could gather from contemporary scientific observations. Further critical evaluation of the individual components and whole medicines is required for more definitive claims.

\section{Conclusions}

Acharya Sushruta says "it is allowed to interpret concepts of a discipline through premises of other sciences to comprehend wider connotations with an ultimate objective of common good" [52]. The present study was an endeavor in this direction. There is evidence from the current data and literature to support the usefulness of adjuvant Ayurveda therapies in modulating cardiac autonomic dysfunction in ischemic stroke. However, further studies that can incorporate more aspects of individualized whole system protocols of Ayurveda 
treatment and in a larger population are definitely warranted to enhance the benefits of the science.

\section{Conflict of Interests}

The authors declare that there is no conflict of interests regarding the publication of this paper.

\section{Acknowledgments}

The authors thank the Central Council for Research in Ayurveda \& Siddha (CCRAS), Department of AYUSH, Ministry of Health \& Family Welfare, Government of India, New Delhi, India, for funding the project. The publication of the paper was largely supported by the clinical research corpus to FRLHT-ITDHST, Bangalore from the Jamsetji Tata Trusts, Mumbai.

\section{References}

[1] J. T. Korpelainen, K. A. Sotaniemi, K. Suominen, U. Tolonen, and V. V. Myllylä, "Cardiovascular autonomic reflexes in brain infarction," Stroke, vol. 25, no. 4, pp. 787-792, 1994.

[2] W. T. Talman, "The central nervous system and cardiovascular control in health and disease," in Clinical Autonomic Disorders, P. A. Low, Ed., pp. 47-60, Lippincott-Raven, New York, NY, USA, 1997.

[3] A. H. Ropper, "Management of the autonomic storm," in Clinical Autonomic Disorders, P. A. Low, Ed., pp. 791-802, Lippincott-Raven, New York, NY, USA, 1997.

[4] S. L. Dawson, B. N. Manktelow, T. G. Robinson, R. B. Panerai, and J. F. Potter, "Which parameters of beat-to-beat blood pressure and variability best predict early outcome after acute ischemic stroke?" Stroke, vol. 31, no. 2, pp. 463-468, 2000.

[5] P. J. Eames, M. J. Blake, S. L. Dawson, R. B. Panerai, and J. F. Potter, "Dynamic cerebral autoregulation and beat to beat blood pressure control are impaired in acute ischaemic stroke," Journal of Neurology Neurosurgery and Psychiatry, vol. 72, no. 4, pp. 467$472,2002$.

[6] N. Lakusic, D. Mahovic, and T. Babic, "Gradual recovery of impaired cardiac autonomic balance within first six months after ischemic cerebral stroke," Acta Neurologica Belgica, vol. 105, no. 1, pp. 39-42, 2005.

[7] A. McLaren, S. Kerr, L. Allan et al., "Autonomic function is impaired in elderly stroke survivors," Stroke, vol. 36, no. 5, pp. 1026-1030, 2005.

[8] T. G. Robinson, S. L. Dawson, P. J. Eames, R. B. Panerai, and J. F. Potter, "Cardiac baroreceptor sensitivity predicts long-term outcome after acute ischemic stroke," Stroke, vol. 34, no. 3, pp. 705-711, 2003.

[9] M. Sykora, J. Diedler, P. Turcani, W. Hacke, and T. Steiner, "Baroreflex: a new therapeutic target in human stroke?" Stroke, vol. 40, no. 12, pp. e678-e682, 2009.

[10] J.-L. Elghozi and C. Julien, "Sympathetic control of shortterm heart rate variability and its pharmacological modulation," Fundamental and Clinical Pharmacology, vol. 21, no. 4, pp. 337347, 2007.

[11] D. H. Barer, J. M. Cruickshank, S. B. Ebrahim, and J. R. A. Mitchell, "Low dose $\beta$ blockade in acute stroke ('BEST' trial): an evaluation," British Medical Journal, vol. 296, no. 6624, pp. 737-741, 1988.
[12] T. Dziedzic, A. Slowik, J. Pera, and A. Szczudlik, "Beta-blockers reduce the risk of early death in ischemic stroke," Journal of the Neurological Sciences, vol. 252, no. 1, pp. 53-56, 2007.

[13] A. Charaka, A. Drdhabala, and A. Chakrapanidatta, The Charaka Samhita of Agnivesha, Munshiram Manoharlal Publishers, Varanasi, India, 1992.

[14] A. R. Gujjar, T. N. Sathyaprabha, D. Nagaraja, K. Thennarasu, and N. Pradhan, "Heart rate variability and outcome in acute severe stroke: role of power spectral analysis," Neurocritical Care, vol. 1, no. 3, pp. 347-353, 2004.

[15] K. Udupa, T. N. Sathyaprabha, J. Thirthalli et al., "Alteration of cardiac autonomic functions in patients with major depression: a study using heart rate variability measures," Journal of Affective Disorders, vol. 100, no. 1-3, pp. 137-141, 2007.

[16] "Heart rate variability: standards of measurement, physiological interpretation and clinical use. Task Force of the European Society of Cardiology and the North American Society of Pacing and Electrophysiology," European Heart Journal, vol. 17, pp. 354-381, 1996.

[17] T. G. Robinson, M. James, J. Youde, R. Panerai, and J. Potter, "Cardiac baroreceptor sensitivity is impaired after acute stroke," Stroke, vol. 28, no. 9, pp. 1671-1676, 1997.

[18] J. Tank, R. M. Baevski, A. Fender et al., "Reference values of indices of spontaneous baroreceptor reflex sensitivity," American Journal of Hypertension, vol. 13, no. 3, pp. 268-275, 2000.

[19] H. S. Kasture, Ayurvediya Panchakarma Vijnana, Shree Baidyanath Ayurveda Bhavana, Nagpur, India, 1997.

[20] B. Patwardhan, "Ayurveda GCP Guidelines: need for freedom from RCT ascendancy in favor of whole system approach," Journal of Ayurveda and Integrative Medicine, vol. 2, no. 1, pp. $1-4,2011$.

[21] J. T. Korpelainen, K. A. Sotaniemi, H. V. Huikuri, and V. V. Myllylä, "Abnormal heart rate variability as a manifestation of autonomic dysfunction in hemispheric brain infarction," Stroke, vol. 27, no. 11, pp. 2059-2063, 1996.

[22] J. T. Korpelainen, H. V. Huikuri, K. A. Sotaniemi, and V. V. Myllylä, "Abnormal heart rate variability reflecting autonomic dysfunction in brainstem infarction," Acta Neurologica Scandinavica, vol. 94, no. 5, pp. 337-342, 1996.

[23] M. Dütsch, M. Burger, C. Dörfler, S. Schwab, and M. J. Hilz, "Cardiovascular autonomic function in poststroke patients," Neurology, vol. 69, no. 24, pp. 2249-2255, 2007.

[24] A. M. Mäkikallio, T. H. Mäkikallio, J. T. Korpelainen, K. A. Sotaniemi, H. V. Huikuri, and V. V. Myllylä, "Heart rate dynamics predict poststroke mortality," Neurology, vol. 62, no. 10, pp. 1822-1826, 2004.

[25] F. Lombardi, "Clinical implications of present physiological understanding of HRV components," Cardiac Electrophysiology Review, vol. 6, no. 3, pp. 245-249, 2002.

[26] A. Malliani, M. Pagani, F. Lombardi, and S. Cerutti, "Cardiovascular neural regulation explored in the frequency domain," Circulation, vol. 84, no. 2, pp. 482-492, 1991.

[27] M. Pagani, F. Lombardi, and S. Guzzetti, "Power spectral analysis of heart rate and arterial pressure variabilities as a marker of sympatho-vagal interaction in man and conscious dog," Circulation Research, vol. 59, no. 2, pp. 178-193, 1986.

[28] D. L. Eckberg, "Sympathovagal balance: a critical appraisal," Circulation, vol. 96, no. 9, pp. 3224-3232, 1997.

[29] D. S. Goldstein, O. Bentho, M.-Y. Park, and Y. Sharabi, "Lowfrequency power of heart rate variability is not a measure of cardiac sympathetic tone but may be a measure of modulation 
of cardiac autonomic outflows by baroreflexes," Experimental Physiology, vol. 96, no. 12, pp. 1255-1261, 2011.

[30] J. P. Moak, D. S. Goldstein, B. A. Eldadah et al., "Supine lowfrequency power of heart rate variability reflects baroreflex function, not cardiac sympathetic innervation," Heart Rhythm, vol. 4, no. 12, pp. 1523-1529, 2007.

[31] F. Rahman, S. Pechnik, D. Gross, L. Sewell, and D. S. Goldstein, "Low frequency power of heart rate variability reflects baroreflex function, not cardiac sympathetic innervation," Clinical Autonomic Research, vol. 21, no. 3, pp. 133-141, 2011.

[32] F. Lombardi and P. K. Stein, "Origin of heart rate variability and turbulence: an appraisal of autonomic modulation of cardiovascular function," Frontiers in Physiology, vol. 2, p. 95, 2011.

[33] B. Auddy, M. Ferreira, F. Blasina et al., "Screening of antioxidant activity of three Indian medicinal plants, traditionally used for the management of neurodegenerative diseases," Journal of Ethnopharmacology, vol. 84, no. 2-3, pp. 131-138, 2003.

[34] S. S. Swathy, S. Panicker, R. S. Nithya, M. M. Anuja, S. Rejitha, and M. Indira, "Antiperoxidative and antiinflammatory effect of sida cordifolia linn. on quinolinic acid induced neurotoxicity," Neurochemical Research, vol. 35, no. 9, pp. 1361-1367, 2010.

[35] A. K. Tiwari, P. V. Srinivas, S. Praveen Kumar, and J. Madhusudana Rao, "Free radical scavenging active components from Cedrus deodara," Journal of Agricultural and Food Chemistry, vol. 49, no. 10, pp. 4642-4645, 2001.

[36] F. Atif, S. Yousuf, and S. K. Agrawal, "S-Allyl L-cysteine diminishes cerebral ischemia-induced mitochondrial dysfunctions in hippocampus," Brain Research, vol. 1265, pp. 128-137, 2009.

[37] B. Ray, N. B. Chauhan, and D. K. Lahiri, "Oxidative insults to neurons and synapse are prevented by aged garlic extract and S-allyl-l-cysteine treatment in the neuronal culture and APPTg mouse model," Journal of Neurochemistry, vol. 117, no. 3, pp. 388-402, 2011.

[38] J. Wattanathorn, J. Jittiwat, T. Tongun, S. Muchimapura, and K. Ingkaninan, "Zingiber officinale mitigates brain damage and improves memory impairment in focal cerebral ischemic rat," Evidence-Based Complementary and Alternative Medicine, vol. 2011, Article ID 429505, 8 pages, 2011.

[39] M. L. Alexandrova, P. G. Bochev, V. I. Markova et al., "Oxidative stress in the chronic phase after stroke," Redox Report, vol. 8, no. 3, pp. 169-176, 2003.

[40] M. L. Alexandrova and P. G. Bochev, "Oxidative stress during the chronic phase after stroke," Free Radical Biology and Medicine, vol. 39, no. 3, pp. 297-316, 2005.

[41] D. Canistro, A. A. Affatato, A. Soleti et al., "The novel radical scavenger IAC is effective in preventing and protecting against post-ischemic brain damage in Mongolian gerbils," Journal of the Neurological Sciences, vol. 290, no. 1-2, pp. 90-95, 2010.

[42] T. Field and M. Diego, "Vagal activity, early growth and emotional development," Infant Behavior and Development, vol. 31, no. 3, pp. 361-373, 2008.

[43] K. Takamoto, S. Sakai, E. Hori et al., "Compression on trigger points in the leg muscle increases parasympathetic nervous activity based on heart rate variability," Journal of Physiological Sciences, vol. 59, no. 3, pp. 191-197, 2009.

[44] D. Lucini, M. Malacarne, N. Solaro, S. Busin, and M. Pagani, "Complementary medicine for the management of chronic stress: superiority of active versus passive techniques," Journal of Hypertension, vol. 27, no. 12, pp. 2421-2428, 2009.

[45] K. Kukkonen-Harjula and K. Kauppinen, "Health effects and risks of sauna bathing," International Journal of Circumpolar Health, vol. 65, no. 3, pp. 195-205, 2006.
[46] Y. Nagashima, H. Oda, M. Igaki et al., "Application of heatand steam-generating sheets to the lumbar or abdominal region affects autonomic nerve activity," Autonomic Neuroscience, vol. 126-127, pp. 68-71, 2006.

[47] H. Yasui, K. Takamoto, E. Hori et al., "Significant correlation between autonomic nervous activity and cerebral hemodynamics during thermotherapy on the neck," Autonomic Neuroscience, vol. 156, no. 1-2, pp. 96-103, 2010.

[48] The Ayurvedic Formulary of India, Department of Indian Systems of Medicine and Homeopathy, Ministry of Health and Family Welfare, Government of India, New Delhi, India, 1st edition, 2003.

[49] A. Vagbhata, A. Arunadatta, and A. Hemadri, Ashtanga Hridaya, Krishnadas Academy, Varanasi, India, 1995.

[50] R. Sharma and S. S. Sharma, Sahasrayogam, Dakshina Prakashana, Hyderabad, India, 1st edition, 1989.

[51] A. Govinda Das Sen, Bhaishajya Ratnavali, Khemraj Srikrishnadas Prakashan, Bombay, India, 2001.

[52] A. Sushruta, A. Dalhanacharya, and A. Gayadasacharya, Sushruta Samhita, Krishnadas Academy, Varanasi, India, 1998. 


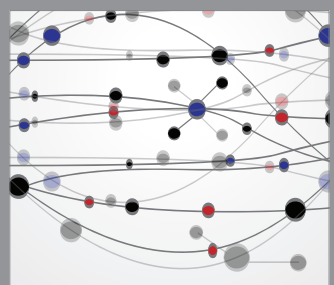

The Scientific World Journal
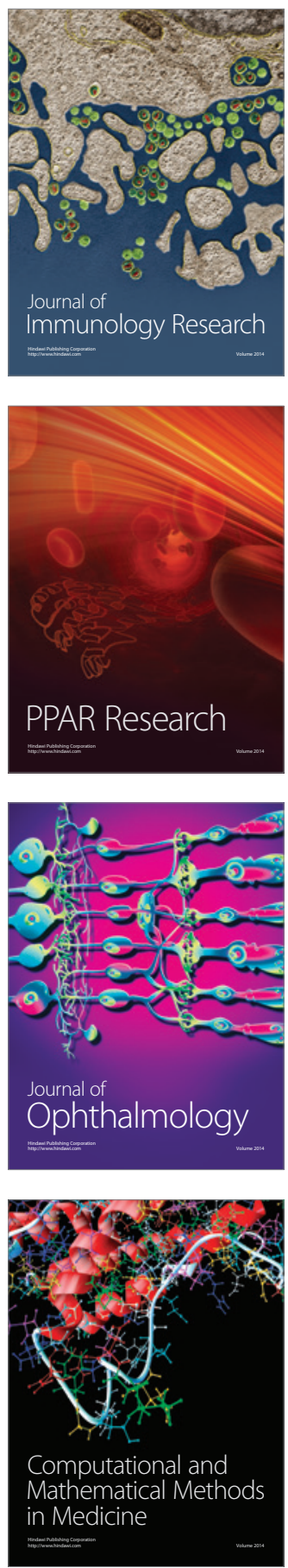



Gastroenterology

Research and Practice
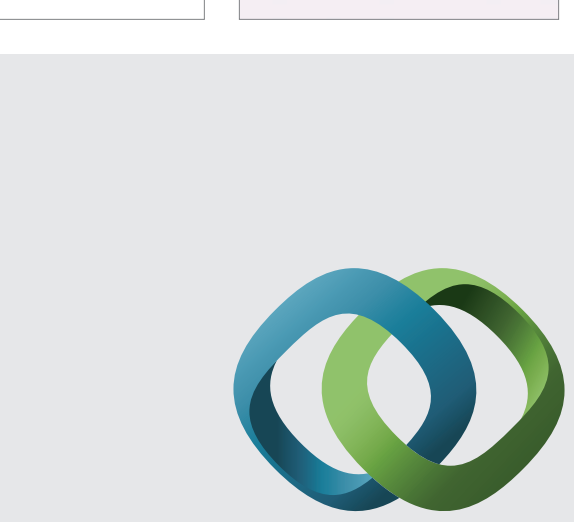

\section{Hindawi}

Submit your manuscripts at

http://www.hindawi.com
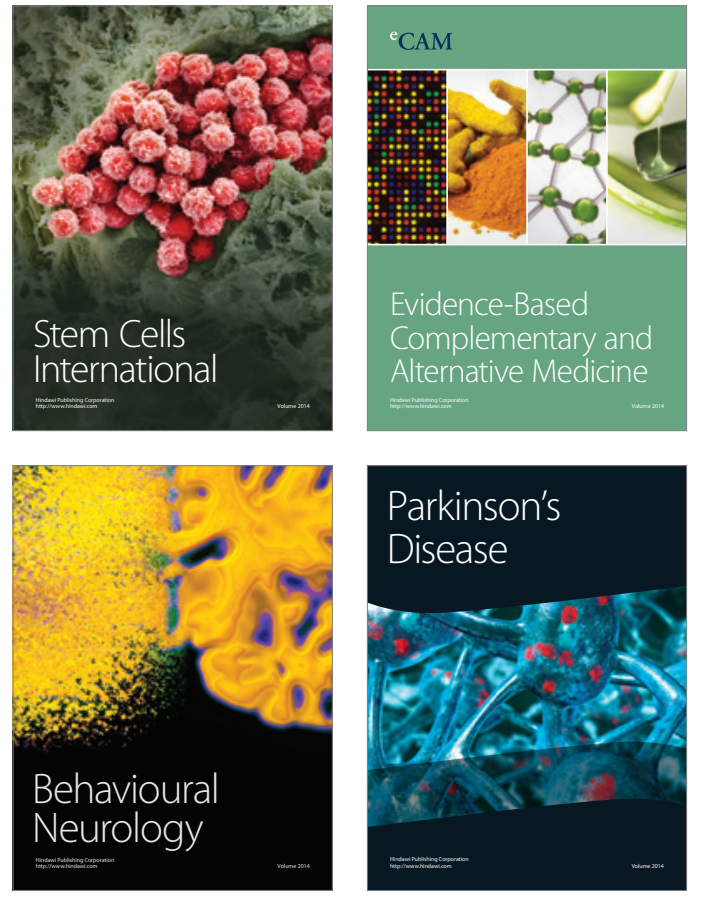
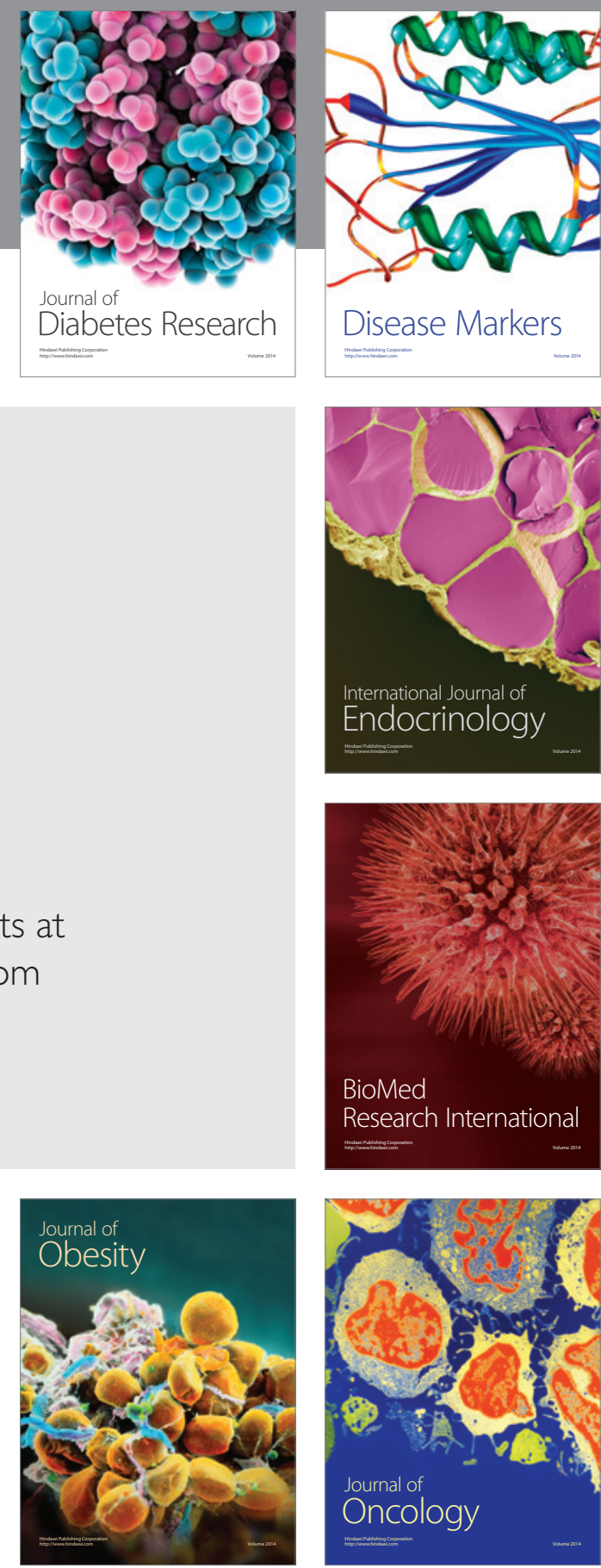

Disease Markers
